\title{
Multiple Intracerebral Arteriovenous Malformations in Deep Structure -Case Report-
}

\author{
Eiji KoHMURA, Takuyu TAKI and Tsuneo TANIOKA \\ Department of Neurosurgery, Iseikai Hospital, Osaka
}

\begin{abstract}
The authors describe a case of multiple intracerebral arteriovenous malformations (AVMs) in a 23year-old male with two distinct, deep-seated AVMs. One was located in the left basal ganglia, which had bled twice, and the other in the splenium. They were removed separately. He recovered satisfactorily with only a mild dysphasia. The authors emphasize that the therapeutic principle for multiple AVMs is the same as that for a solitary AVM. Multiplicity alone does not dictate the operability. Dissection just adjacent to the nidus and direct coagulation of an AVM are the indicated techniques, especially in cases of deep-seated AVMs in order to reduce postoperative neurological deficit.
\end{abstract}

Key words: central nervous system, multiple arteriovenous malformations, deep-seated lesion

\section{Introduction}

Multiple cerebral arteriovenous malformations (AVMs) are rarely encountered, while cerebral aneurysm is frequently multiple. A cooperative study, ${ }^{31}$ for example, disclosed only 3 cases $(0.7 \%)$ of multiple AVMs in 453 AVMs.

In this paper we report a rare case of two deepseated AVMs in different locations.

\section{Case Report}

A 23-year-old male was admitted to our department because of sudden, severe headache and vomiting. He had had a left intracerebral hematoma 4 years prior to admission. Neurological examination revealed mild right hemiparesis, dysphasia, and stiff neck. Precontrast computed tomographic (CT) scans showed a small hematoma in the left basal ganglia with intraventricular hemorrhage. A small low-density area found adjacent to the hematoma was considered to be due to the previous hemorrhage (Fig. 1 upper). Postcontrast CT scans showed tortuous enhanced lesions in the left basal ganglia and the dilatation of the internal cerebral vein and the great vein of Galen (Fig. 1 lower). A right ventriculostomy was emplaced for 10 days. Cerebral angiograms demonstrated two distinct AVMs (Fig. 2). One was located in the left basal ganglia and supplied by the left lenticulostriate and posterior thalamoperforating arteries. It drained into the left internal cerebral vein. The other was located in the splenium and supplied by the anterior and posterior pericallosal arteries, and drained into the vein of Galen.

Two weeks later, his neurological status improved. He was fully conscious with only a mild dysphasia. His right hemiparesis completely disappeared. We decided to operate because he was still young and this was the second bleeding.

First, the AVM in the left basal ganglia, responsible for the bleeding, was approached transcortically. The old hematoma cavity was entered and the nidus coagulated gradually under continuous irrigation. The feeding arteries were divided stepwise just adja-

Received November 17, 1989; Accepted March 19, 1990

Authors' present addresses: E. Kohmura, M.D., Department of Neurosurgery, Osaka University Medical School, Osaka, Japan; T. Taki, M.D., Department of Neurosurgery, Toyonaka City Hospital, Toyonaka, Osaka, Japan. 

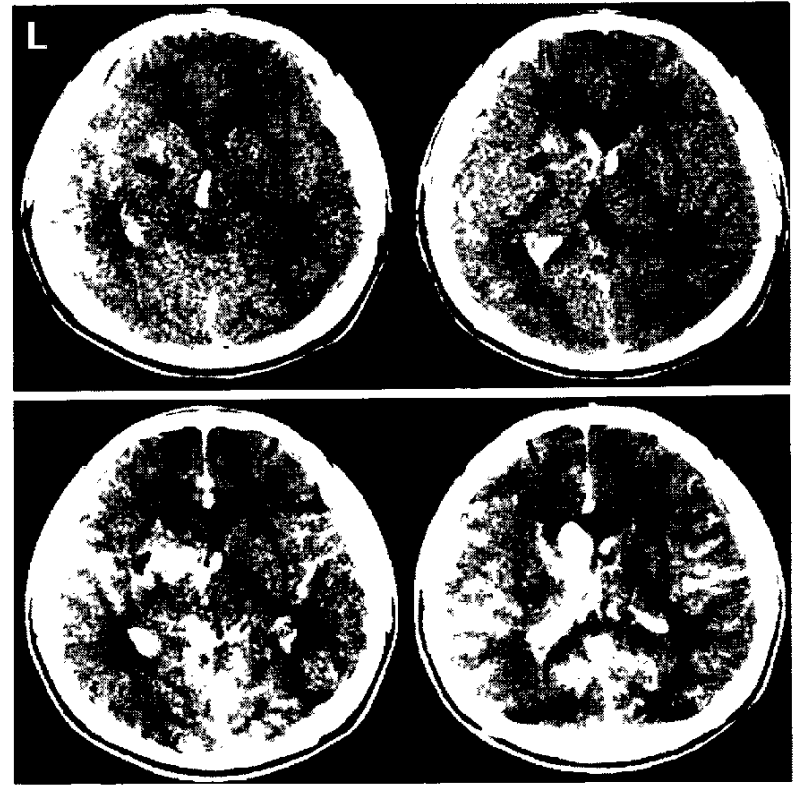

Fig. 1 Preoperative CT scans. upper: Precontrast CT scans, showing a small hematoma in the left caudate nucleus and an intraventricular hemorrhage. A small low-density area in the left globus pallidus is considered to be due to a previous hemorrhage. lower: Postcontrast CT scans, showing a markedly enhanced lesion in the left basal ganglia and dilatation of internal cerebral vein and great vein of Galen.

cent to the nidus, in order to minimize additional brain damage. After the AVM was removed from the caudate nucleus and the globus pallidus, the third ventricle was entered. The internal cerebral vein was found still red. Although a part of the nidus remained in the thalamus, we decided not to go further in order to avoid additional neurological deficits.

He awoke immediately after the operation with a right hemiparesis and a severe dysphasia. In a few days, however, the hemiparesis disappeared completely and the dysphasia improved markedly. Cerebral angiograms showed the greater part of the AVM in the left basal ganglia had disappeared with preservation of the lenticulostriate arteries. A small residual nidus was supplied by the posterior thalamoperforating arteries. The splenial AVM showed no change.

Seven weeks after the first operation the splenial AVM was operated on. The head was elevated in the supine position and a left occipital craniotomy was performed. The left occipital lobe was retracted laterally and the AVM was approached through the interhemispheric space. The nidus was coagulated and the feeding arteries were divided. After the draining vein discolored to blue, it was divided and the AVM was removed.

The postoperative course was uneventful. Postoperative angiograms revealed a small residual nidus supplied by the posterior thalamoperforating arteries (Fig. 3). A postoperative CT scan showed a small enhanced lesion in the left thalamus (Fig. 4).

He was discharged with only a mild dysphasia. At 1 year after surgery his neurological status was stable and he returned to his previous work.

\section{Discussion}

Multiple intracranial AVMs have rarely been
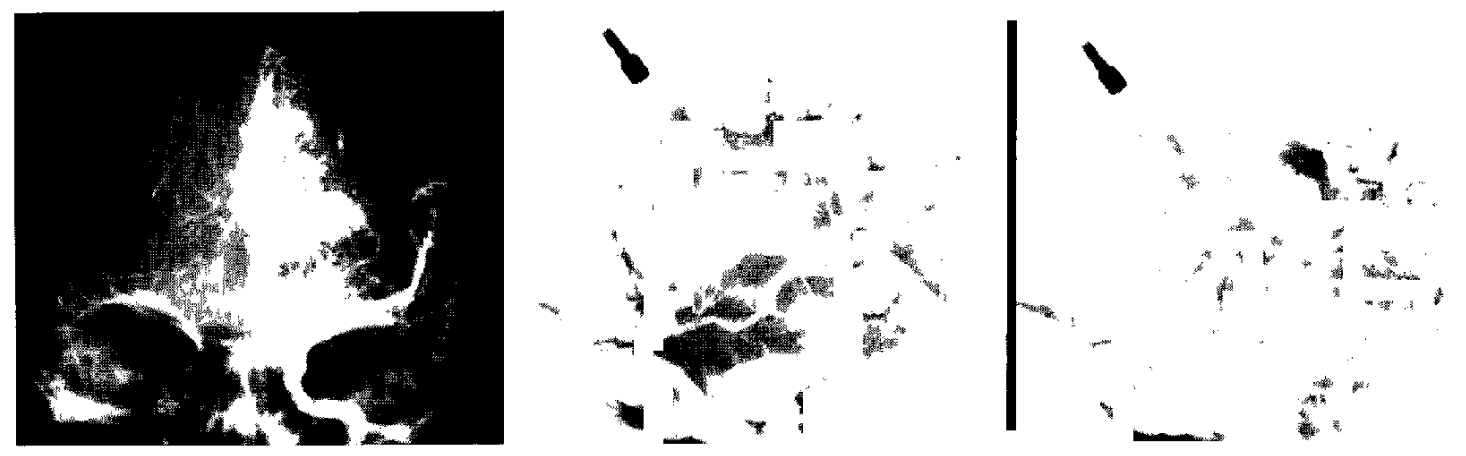

Fig. 2 Preoperative cerebral angiograms. left, center: Left carotid angiograms, anteroposterior (left) and lateral (center) views, showing an AVM in the left basal ganglia supplied from the lenticulostriate arteries and another AVM in the splenium supplied from the pericallosal artery. right: Left vertebral angiogram, lateral view, revealing an AVM in the basal ganglia which is fed by the posterior thalamoperforating arteries and the medial posterior choroidal artery. A splenial AVM supplied from the posterior pericallosal artery is also shown. 

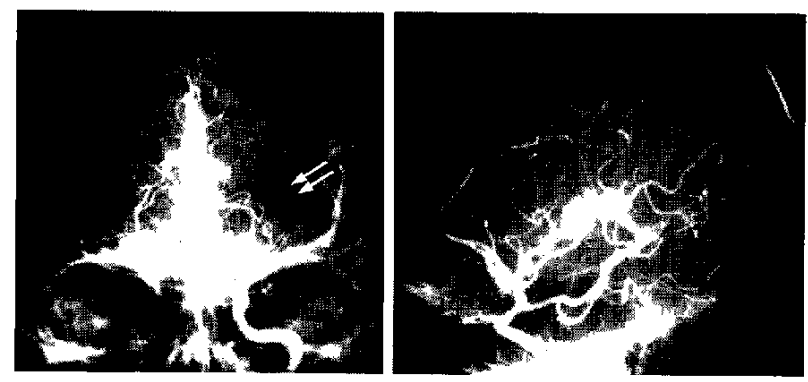

Fig, 3 Cerebral angiograms, anteroposterior (left) and lateral (right) views, after the second operation. Most of the AVM was removed from the left basal ganglia with preservation of the lenticulostriate arteries (arrows). A small residual nidus supplied by the posterior thalamoperforating arteries can be seen.

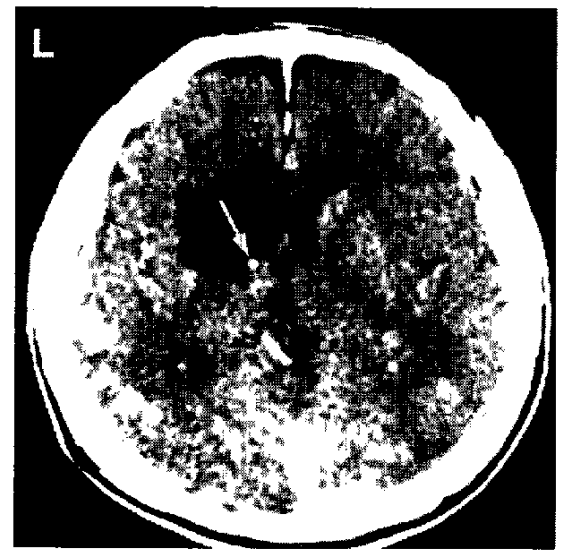

Fig. 4 Postoperative postcontrast CT scan demonstrating a small enhanced lesion in the left thalamus (arrow).

reported..$^{1,4-7,91}$ McCormick ${ }^{21}$ described the incidence of multiple angiomas as $6 \%$ of his autopsy cases. However, his series included not only AVMs but also all other angiomas. It is difficult to know the accurate incidence of multiple AVMs. A large clinical series should be referenced. Perret and Nishioka ${ }^{3}$ reported only 3 cases $(0.7 \%)$ with multiple AVMs in their 453 AVMs in a cooperative study. Yasargil ${ }^{8)}$ reported 15 patients $(3.0 \%)$ with multiple AVMs in his 500 AVM cases, which may be a collection of selected difficult cases. There was no preferred location. Yasargil described the treatment of multiple AVMs as essentially the same as for solitary lesions. Tada et al. ${ }^{6)}$ emphasized that the size and the structures involved are more important factors in the prognosis than the multiplicity of the lesions. For multiple AVMs with bleeding, the one responsible for the bleeding is to be treated with priority.

Many factors should be considered before attempting to remove AVMs. The age of the patient as well as the general and neurological status are important factors. The size and the location of the AVM, pattern of the blood supply and draining, and history of bleeding are also important. In our case, the AVMs were located in the deep cerebral structures, one in the left basal ganglia and the other in the splenium. These locations presented us with therapeutic difficulties. Although surgical indication for deep-seated AVM is still controversial, it should be operated on when the patient is young, his neurological condition is satisfactory, the AVM has bled repeatedly, and the AVM is not large.

To remove AVMs in the basal ganglia, transcortical, trans-Sylvian, and interhemispheric approaches can be considered. We have chosen the transcortical route. In our case the AVM could be approached directly by this route, because it extended rather in the coronal direction. Moreover, there was a small cavity caused by the previous hemorrhage, which allowed us to dissect the AVM with minimal damage to the surrounding important tissue. For deep-seated AVMs the dissection just adjacent to the nidus is unavoidable to prevent in jury to surrounding important tissue. Any small perforating artery adjacent to the AVM should be preserved. A useful technique for this purpose is direct coagulation of the nidus. If the AVM is not large, the nidus can be coagulated stepwise under continuous irrigation. Although profuse bleeding may occur during coagulation of the nidus, such bleeding can be controlled with gentle compression of the nidus. By coagulating the nidus one can obtain more space with minimal brain retraction.

In our case, a postoperative angiogram confirmed a small residual nidus in the left thalamus, but the family refused further surgery. Although we consider, in general, that any residual nidus should be removed, we decided to follow-up on this patient. Further removal in the thalamus seemed too risky. Other therapeutic possibilities, such as stereotactic radiosurgery, ${ }^{5)}$ might be considered.

\section{References}

1) Hanieh A, Blumbergs PC, Carney PG: Multiple cerebral arteriovenous malformations associated with soft-tissue vascular malformations. $J$ Neurosurg 54: 670-672, 1981

2) McCormick WF: The pathology of angiomas, in Fein JM, Flamm ES (eds): Cerebrovascular Surgery, vol 4. 
New York, Springer, 1985, pp 1073-1095

3) Perret $G$, Nishioka $H$ : Report on the cooperative study of intracranial aneurysms and subarachnoidal hemorrhage: Section 4. Arteriovenous malformations. Analysis of 545 cases of craniocerebral arteriovenous malformations and fistulae reported to the cooperative study. $J$ Neurosurg 25: 467-490, 1966

4) Schlachter LB, Fleischer AS, Faria MA Jr, Tindall GT: Multifocal intracranial arteriovenous malformations. Neurosurgery 7: 440-444, 1980

5) Steiner L: Radiosurgery in cerebral arteriovenous malformations, in Fein JM, Flamm ES (eds): Cerebrovascular Surgery, vol 4. New York, Springer, 1985, pp 1161-1215

6) Tada T, Sugita K, Kobayashi S, Watanabe N: Supraand infratentorial arteriovenous malformations with an aneurysmal dilatation: A case report. Neurosurgery
19: $831-834,1986$

7) Tamaki N, Fujita K, Yamashita H: Multiple arteriovenous malformations involving the scalp, dura, retina, cerebrum, posterior fossa: Case report. $J$ Neurosurg 34: 95-98, 1971

8) Yasargil MG: Microneurosurgery, vol $3 \mathrm{~A}$. Stuttgart, Georg Thieme, 1984, pp 165-181

9) Zellen RT, Buchheit WA: Multiple intracranial arteriovenous malformations: Case report. Neurosurgery 17: $88-93,1985$

Address reprint requests to: E. Kohmura, M.D., Department of Neurosurgery, Osaka University Medical School, 1-1-50 Fukushima, Fukushima-ku, Osaka 553, Japan. 